\title{
Overview of studies on the effect of recycled aggregates sourced from tested cylinders on concrete material and structural properties
}

\author{
Hamad Bilal ${ }^{1 *}$ and Dawi $\mathrm{Ali}^{2}$ \\ ${ }^{1}$ American University of Beirut, P.O. Box: 11-0236, Riad El-Solh, 1107 2020, Beirut, Lebanon \\ ${ }^{2}$ CODE Consultants \& Designers sarl, Mina El Hoson, Phoenicia St., Sfeir Bldg., Beirut, Lebanon
}

\begin{abstract}
The paper presents an overview of a two-phase research program that was designed at the American University of Beirut (AUB) to investigate the effect of replacing different percentages of natural coarse aggregates (NCA) with recycled coarse aggregates (RCA) on the properties of the produced concrete. The source of RCA was tested cylinders in batching plants which would help recycling and reusing portion of the waste products of the concrete industry. In the first phase, the fresh and hardened mechanical properties of the produced concrete mix were investigated. The variables were the concrete strength $(28$ or $60 \mathrm{MPa})$ and the percentage replacement of NCA with RCA from crushed tested cylinders [0 (control), 20, 40,60,80, or $100 \%$ ). Normal strength tested cylinders were used as source of the recycled aggregates for the normal strength concrete (NSC) mix and high strength tested cylinders were used for the high strength concrete (HSC) mix. Tests included plastic state slump and hardened state mechanical properties including cylinder compressive strength, cylinder splitting tensile strength, modulus of elasticity, and standard beams flexural strength. The results indicated no significant effect on the slump and around $10 \%$ average reduction in the hardened mechanical properties for both investigated levels of concrete compressive strength. In the second phase, the structural behavior of normal strength concrete (NSC) reinforced concrete beams prepared by replacing different percentages of NCA with RCA from tested concrete cylinders was studied. For each of three modes of failure (flexural, shear, or bond splitting), three beams with different percentages replacement $[0$ (control), 40, or 100 percent] were tested. One replicate was prepared for each beam to validate the test results. Results indicated no significant difference in the ultimate load reached or load-deflection behavior that could be related to the percentage replacement of NCA with RCA.
\end{abstract}

\footnotetext{
* Corresponding author: bhamad@aub.edu.lb
} 


\section{Introduction}

Construction and demolition wastes (CDW) are produced worldwide due to the development of cities and/or due to emergencies such as earthquakes and wars. CDW can be defined as a mixture of surplus materials generated during new construction, renovation, and demolition of buildings, roads, bridges, and other structures. the boost in construction activities that led to the annual production of 500,000 ton of CDW in the city of Beirut by the demolition of a large number of existing old facilities either because of the limited land for new development or due to the fact the existing buildings are structurally defected. Another source of cementitious based CDW comes from quality control procedures. Common practices require the sampling and laboratory testing of standard cylinders. These tested cylinders constitute a significant portion of the dumped CDW. A typical batching plant generates between 20 and 80 tons of waste per month.

The accumulation of such CDWs has detrimental impact on the environment especially in developing countries that lack plans and methods to manage, handle, dispose, recycle, and reuse dismantled waste properly. Therefore, the incorporation of the cementitious portions of CDW as recycled concrete aggregates (RCA) in concrete mixes has become a commonly proposed solution that would help the preservation of natural resources, alleviation of construction waste disposal problem with its negative environmental impact, and production of a sustainable and green concrete material.

In 2004, the Federal Highway Association (FHWA) in USA reported that 38 States recycle concrete as an aggregate base for roadways and 11 States incorporate recycled aggregates into newly produced PCC mixes. States that use RAC have reported that concrete with RCA equally performs as the conventional natural aggregate concrete (NAC) prepared with crushed limestone aggregates.

The American Concrete Institute (ACI) Committee 555 report stated that the recycling of concrete is a relatively simple process [1]. After the removal of non-cementitious component, RCA can be produced by breaking and crushing existing concrete into a material with a specified size and quality. The quality of RCA is very dependent on the quality of the recycled material used.

\section{Previous research}

\subsection{Research on mechanical properties of RAC}

Many papers are reported in the literature on research conducted on the mechanical properties of RAC. Nagataki et al. investigated the effect of several physical properties of recycled aggregates including the structure and amount of adhered mortar on the performance of recycled aggregate concrete [2]. The results indicated that all possible variations in the recycled aggregate properties should be taken into account in designing durable recycled aggregate concretes.

In 2007, Rahal compared several mechanical properties of RAC and NAC made with the same mix proportions to achieve a nominal concrete strength between 25 and $30 \mathrm{MPa}$ [3]. Rahal concluded that at 28 days, the cube and cylinder compressive strength and the indirect shear strength of RAC were on the average $10 \%$ less than those of NAC. The reduction in the modulus of elasticity was only $3 \%$.

Etxeberria et al. investigated RAC produced using four different percentage replacements of NCA with RCA used in the wet unsaturated condition: 0, 25, 50 and 100\% [4]. Results indicated lower modulus of elasticity values when recycled coarse aggregates were used. 
Yang et al. studied the influence of the type and percentage replacement of NCA with RCA on concrete properties [5]. Test results showed that the fresh and hardened properties of concrete containing recycled aggregates were dependent on the relative water absorption of aggregates. In addition, the modulus of rupture and modulus of elasticity of RAC were lower than the design equations specified in ACI Building Code [6] when the relative water absorption of aggregates was above $2.5 \%$.

Recently in 2012, Ahmed reported the effect of introducing RCA originating from CDW in West Australia on the properties of concrete [7]. The percentage replacements by weight of NCA with RCA were 25, 50, 75 and $100 \%$. The results indicated increase in the mechanical properties of concrete made with $25 \%$ replacement recycled aggregates. RAC containing $40 \%$ replacement of cement with fly ash had greater compressive strength at old ages and lower indirect tensile and flexural strengths at all ages.

\subsection{Research on structural behaviour of RAC}

In 2001, Han et al. reported on tests of the shear capacity of twelve simply supported beams cast with different percentage replacements of NCA with RCA [8]. Variables in the test included the span to depth ratio $(1.5,2,3$, and 4); the type of aggregates that included natural, washed recycled and non-washed recycled aggregates; and the amount of shear reinforcement used in the specimens $(0,0.089,0.507$, and 0.823 percent). It was found that the ACI Building Code equations overestimated the shear capacity of RAC beams with span/depth ratio greater than 3 .

In 2009, Gholamreza el al. published the results of flexural strength tests of RAC beams [9]. Variables included the percentage replacement of NCA with RCA, and the flexural reinforcement percentage. The results indicated comparable flexural strength when using recycled aggregates and proportioning them according to the EMV method. It was also found that ACI Building Code equations over-estimated the modulus of rupture.

Sun-Woo Kim et al. tested 144 pullout specimens to investigate the influence of RCA on the bond behavior of deformed bars in concrete [10]. The variables included: the aggregate size, percentage replacement of NCA with RCA, the reinforcing bar direction (vertical and horizontal), and the reinforcing bar location [75 and $225 \mathrm{~mm}$ (2.95 and 8.86 in.) from the bottom]. Results showed that for the same percentage replacement of NCA with RCA, the bond strength increased while decreasing the maximum recycled concrete aggregate size. Results also showed that the increase in the percentage replacement had a negative impact on the compressive strength. The top-bar effect was very significant with bottom cast bars performing better than top cast bars at all ages.

\section{Research significance}

This paper gives an overview of an extensive two-phase research program that was conducted at the American university of Beirut (AUB). The objective of the first phase was to investigate the effect of replacing different percentages NCA with RCA on the plastic and hardened mechanical properties of the produced concrete, whereas the objective of the second phase was to study the effect on the structural behavior of reinforced concrete members. The significance of the research is two-fold. First is the source of RCA which was tested cylinders in batching plants thus helping the recycling and reusing of portion of the waste products of the concrete industry and mitigating its negative environmental impact. Another reason for significance is the investigation of the difference in flexure, shear, and bond splitting structural behavior between NAC and RAC beam elements. No single previous study covered all three structural modes in one research project. 


\section{Experimental program and test results}

\subsection{Phase 1}

In the first phase, six normal strength concrete (NSC) and six high strength concrete (HSC) mixes were prepared. The nominal intended concrete compressive strength of the mixes was $28 \mathrm{MPa}$ for the NSC mixes and $60 \mathrm{MPa}$ for the HSC mixes. Normal strength tested cylinders were used as source of the recycled aggregates for the NSC mixes and high strength tested cylinders were used for the HSC mixes. The six mixes of each strength type were identical except for the percentage replacement of NCA with RCA. Replacement values of $0,20,40,60,80$, and 100 percent were tested. The mixes are referred to as R0 (Control mix), R20, R40, R60, R80, and R100, respectively.

Natural sand with particle sizes ranging from 0.075 to $1.18 \mathrm{~mm}$ was used in all NSC and HSC mixes. All used natural coarse aggregates (NCA) were from the same source. Also all recycled coarse aggregates were the waste product of regular tests conducted at a specific batching plant on NSC and HSC mixes prepared and delivered to the different construction sites. All coarse aggregates, natural and recycled, were washed from dust material prior to oven drying. The mix design was performed in accordance with Absolute Volume Method stipulated in the ACI Committee 211 Standard: ACI 211.1-91 [11]. The mixes were designed and batched to meet the consistency values of $100 \mathrm{~mm}$ for NSC and $200 \mathrm{~mm}$ for HSC. The water/cement ratio prior to water correction due to absorption was 0.57 for NSC and 0.33 for HSC. No admixtures were used in the NSC mixes, however a high performance concrete admixture based on Polycarboxylate Ether was used in the HSC mixes.

The actual slump test results indicated that replacement of NCA with different percentages of RCA had no negative impact on consistency of the concrete mix. This could be related to the fact that all aggregates were batched at SSD conditions regardless of their type. The slump values ranged between 90 and $160 \mathrm{~mm}$ for the NSC mixes and between 180 and $210 \mathrm{~mm}$ for the HSC mixes.

For the NSC mixes, the replacement of different percentages of NCA with RCA led to an average reduction of $9.8 \%$ in the ultimate compressive strength. The trends of compressive strength gain over age were similar for all six mixes regardless of the percentage replacement of NCA with RCA. The average reduction in the splitting tensile strength for all six mixes was $13.5 \%$ and was similar for all percentage replacements of NCA with RCA. Moreover, the average reduction in the modulus of elasticity was $11 \%$. Whereas the reduction was only $3.52 \%$ for the 20 percent replacement mix, close to what was reported by Rahal [3], it jumped above $10 \%$ for the other larger replacement percentages mixes. The average reduction in the flexural strength for the six mixes relative to the control mix with no RCA was $9.2 \%$, and similar to the splitting tensile strength tests the reduction in flexural strength relative to the control mix was almost consistent for all percentage replacement values.

For the six HSC mixes, the average reduction in the concrete compressive strength relative to the control mix was $11 \%$, similar to the value for the NSC mixes which was $9.8 \%$. Similar to the NSC mixes, the trends of compressive strength gain over age were similar for all six mixes. Whereas the 28-days strength of the control mix (58 MPa) was very close to the targeted design value of $60 \mathrm{MPa}$, the compressive strength values of the mixes with different percentage replacement of NCA with RCA ranged from 49.7 to 52.7 $\mathrm{MPa}$. This proves that a compressive strength value above $50 \mathrm{MPa}$ is achievable for RAC.

The average reductions in the splitting tensile strength and the flexural strength relative to the control mix were 10.3 and $10.8 \%$. For the NSC mixes the value for the splitting tensile strength was slightly higher $(13.5 \%)$ but the value for the flexural strength $(9.2 \%)$ 
was slightly lower. Similar to the NSC mixes, the reductions in the splitting tensile strength and the flexural strength were comparable for all percentage replacements of NCA with RCA. Finally, the average reduction in the modulus of elasticity was $11.3 \%$. The reduction was only $5.01 \%$ for the 20 percent replacement mix, close to what was reported by Rahal for NSC mixes [3], but ranged from 9.09 to $14.66 \%$ for the other larger replacement percentages mixes.

\subsection{Phase 2}

Based on the results of the first phase, 18 reinforced concrete beams were tested in three series each with different percentage replacement of NCA with RCA sourced from tested and crushed concrete cylinders [0(control), 40, or 100\%]. The intended concrete strength used was $28 \mathrm{MPa}(4,000 \mathrm{psi})$. In each series, two replicate beams were tested to fail in flexure (Flexure beams), two to fail in shear (Shear beams), and two beams were tested to fail in bond splitting of the concrete cover in the splice region (Bond beams). Replicates were used to validate the test results.

Section details of the different types of beams are shown in Figures 1, 2, and 3. Plastic spacers between the steel cages and the form sides were used to guarantee side and bottom covers of $30 \mathrm{~mm}$. In the shear beams, the spacing of the stirrups in the shear region was large enough to ensure shear failure. In the bond beams, the bottom reinforcing bars were spliced $305 \mathrm{~mm}$ at the middle of the beam. This splice length was designed to develop a steel stress less than yield to ensure bond splitting mode of failure. The beams were tested in positive bending. The loading system was designed to produce a constant moment region in the middle of the beam specimen. A view of the test setup is shown in Figure 4. The loads were applied monotonically in increments of $10 \mathrm{kN}$; i.e. the testing protocol was load control and not displacement control.

The beams are identified by a four-part notation system (refer to Table 1). The first part indicates the concrete strength of the mix ( $\mathrm{N}$ for normal strength). The second part specifies the mode of failure (F for flexure, $\mathrm{S}$ for shear, and $\mathrm{B}$ for bond splitting). The third part designates the type of concrete mix used in casting the beam specimen $(\mathrm{N}$ for natural aggregates with 0 percentage replacement, 40 for 40 percent replacement of NCA with RCA, and 100 for 100 percent replacement), whereas the fourth part designates the listing number of the two replicates ( 1 or 2 ).

Test results of all 18 beams are presented in Table 1 . The listed data includes the ultimate load reached and the corresponding mid-span deflection. Values of ultimate loads of replicate beams are within $10 \%$. Also listed in Table 1 are the concrete compressive strength values at the day of testing. The latter indicate that relative to the batch with no RCA, mixes with $40 \%$ and $100 \%$ replacement percentages of NCA with RCA had reductions in the compressive strength amounting to 9 and $11.7 \%$, respectively. The average ultimate loads of replicate beams in each series of a given mode of failure are listed in Table 2 along with ratios of ultimate loads relative to the control beam with no replacement of NCA with RCA. The average ultimate loads are also shown in Figure 5.

Although the concrete compressive strength values of the $40 \%$ and $100 \%$ replacement flexural beams were less than that of the control mix, however the maximum flexural loads of these specimens were slightly larger than that of the control beam. Also, the loaddeflection histories were almost identical. Thus, the replacement of even $100 \%$ of NCA with RCA did not adversely affect the flexural strength of the beam specimen. 

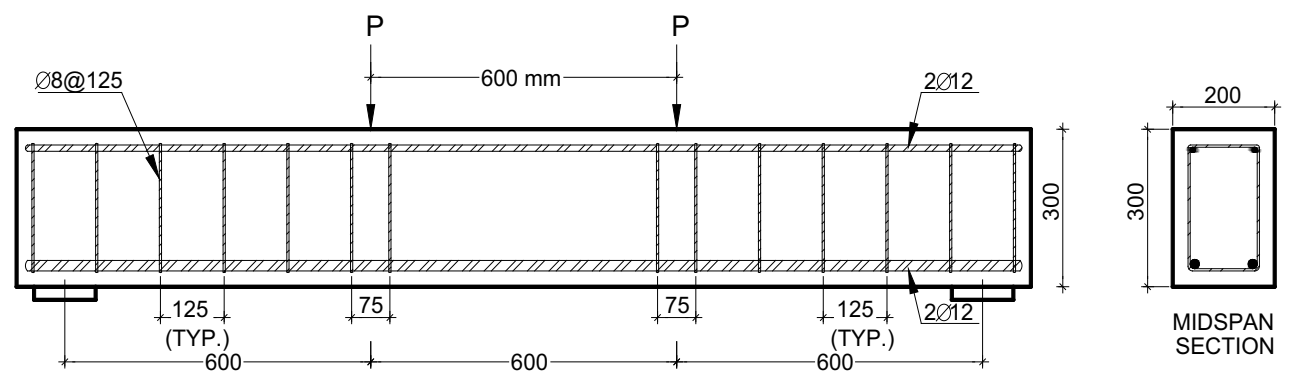

Fig. 1. Flexural beams; all dimensions are in $\mathrm{mm}$.
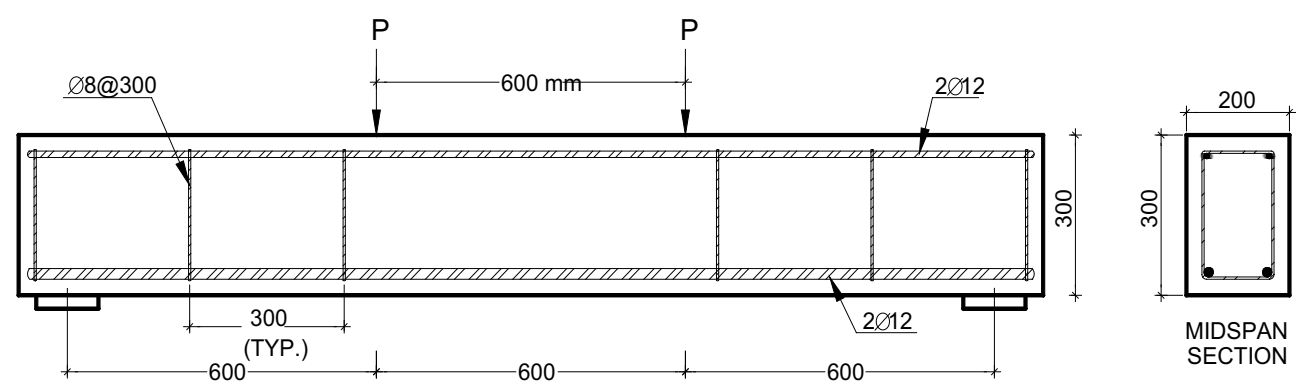

Fig. 2. Shear beams; all dimensions are in $\mathrm{mm}$.
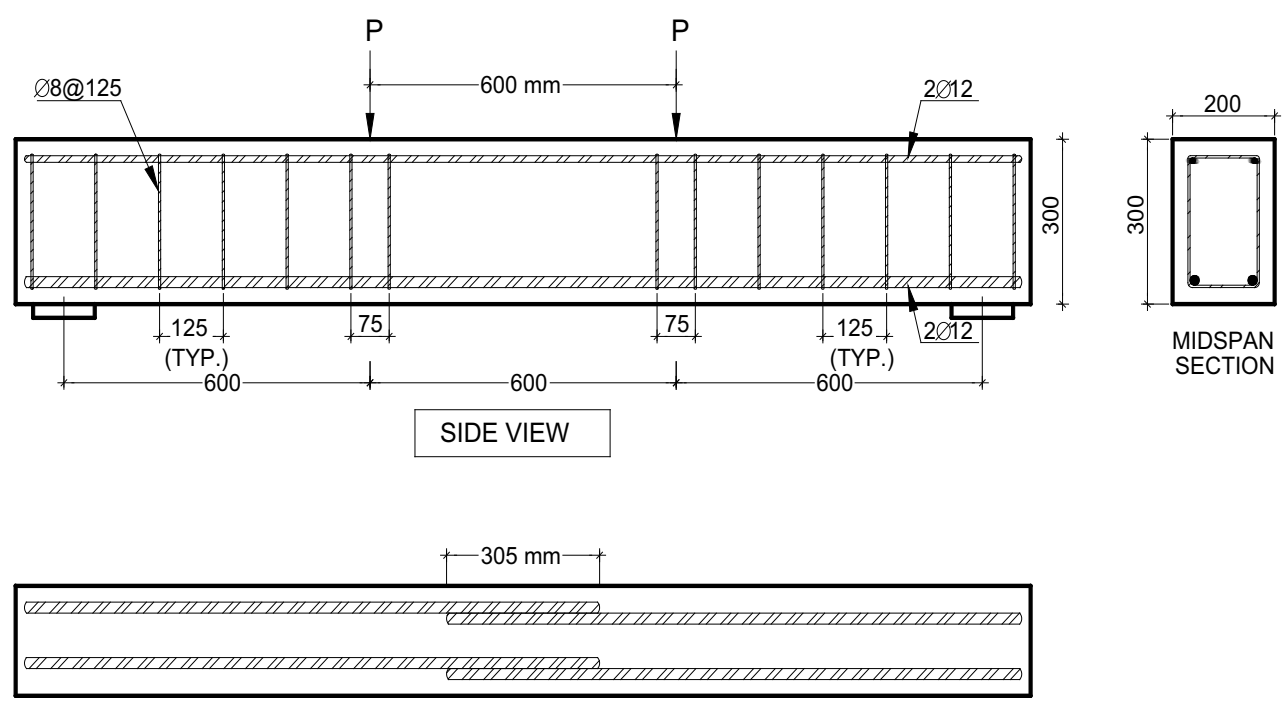

BOTTOM VIEW

Fig. 3. Bond beams; all dimensions are in $\mathrm{mm}$. 


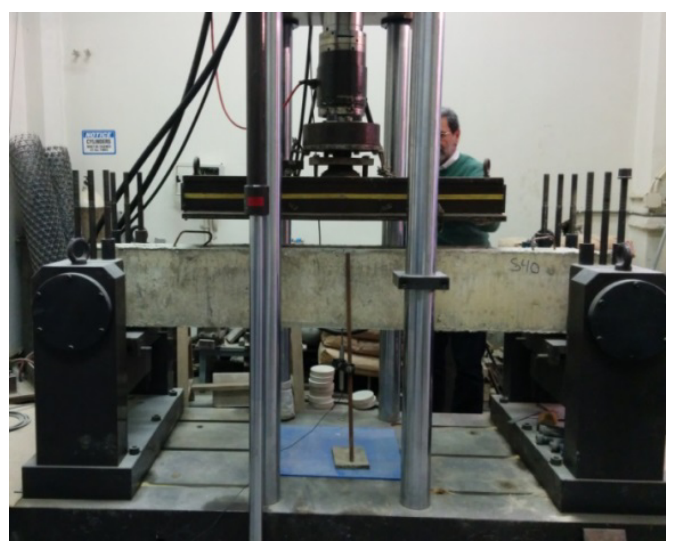

Fig. 4. View of the test setup.

Table 1. Ultimate loads and corresponding centerline deflections.

\begin{tabular}{|c|l|c|c|c|}
\hline \multirow{4}{*}{ Series } & \multicolumn{1}{|c|}{$\begin{array}{c}\text { feam } \\
\text { Notation }\end{array}$} & $\begin{array}{c}\text { f'c } \\
\text { At day of } \\
\text { testing } \\
\text { (MPa) }\end{array}$ & Pmax & $\begin{array}{c}\text { Mid-span } \\
\text { deflection } \\
\text { At Pmax } \\
\text { (mm) }\end{array}$ \\
\hline \multirow{4}{*}{$\begin{array}{c}\text { Series 1 } \\
\text { Flexural Beams }\end{array}$} & NFN-1 & 33.4 & 199.2 & 7.1 \\
\cline { 2 - 5 } & NFN-2 & 33.4 & 194.3 & 8.2 \\
\cline { 2 - 5 } & NF40-1 & 30.7 & 204.2 & 9.1 \\
\cline { 2 - 5 } & NF40-2 & 30.7 & 223.1 & 9.4 \\
\cline { 2 - 5 } & NF100-1 & 29.5 & 240.8 & 11.5 \\
\cline { 2 - 5 } & NF100-2 & 29.5 & 213.6 & 12.1 \\
\hline \multirow{4}{*}{$\begin{array}{c}\text { Series 2 } \\
\text { Shear Beams }\end{array}$} & NSN-1 & 33.4 & 155.2 & 6.1 \\
\cline { 2 - 5 } & NSN-2 & 33.4 & 170.5 & 7.5 \\
\cline { 2 - 5 } & NS40-1 & 30.7 & 159.1 & 7.3 \\
\cline { 2 - 5 } & NS40-2 & 30.7 & 166.6 & 6.8 \\
\cline { 2 - 5 } & NS100-1 & 29.5 & 158.4 & 8.5 \\
\cline { 2 - 5 } & NS100-2 & 29.5 & 160.2 & 7.8 \\
\hline \multirow{4}{*}{$\begin{array}{c}\text { Series 3 } \\
\text { Bond Beams }\end{array}$} & NBN-1 & 33.4 & 118.1 & 4.3 \\
\cline { 2 - 5 } & NBN-2 & 33.4 & 120.2 & 4.5 \\
\cline { 2 - 5 } & NB40-1 & 30.7 & 140.6 & 3.8 \\
\cline { 2 - 5 } & NB40-2 & 30.7 & 135.2 & 4.2 \\
\cline { 2 - 5 } & NB100-1 & 29.5 & 120.2 & 5.2 \\
\cline { 2 - 5 } & NB100-2 & 29.5 & 122.2 & 5.3 \\
\hline
\end{tabular}

* NFN-1: The first "N" stands for Normal strength concrete, "F" stands for flexural mode of failure; the second "N" stands for natural coarse aggregate or $0 \%$ replacement of NCA with RCA; and " 1 " stands for the first of two identical replicates.

+ NS40-2: "N" stands for Normal strength concrete, "S" stands for shear mode of failure; " 40 " stands for $40 \%$ replacement of NCA with RCA; and " 2 " stands for the second of two identical replicates.

++ NB100-1: "N" stands for Normal strength concrete, "B" stands for bond splitting mode of failure; " 100 " stands for $100 \%$ replacement of NCA with RCA; and "1" stands for the first of two identical replicates. 
Table 2. Average ultimate loads for the replicate beams in the three series.

\begin{tabular}{|c|c|c|c|}
\hline \multirow{2}{*}{ Series } & Beam Notation & $\begin{array}{c}\text { Average } \\
\text { ultimate load } \\
\mathbf{( k N )}^{*}\end{array}$ & $\begin{array}{c}\text { Ratio relative to } \\
\text { the control } \\
\text { beam }^{* * *}\end{array}$ \\
\hline \multirow{2}{*}{$\begin{array}{c}\text { Series 1 } \\
\text { Flexural Beams }\end{array}$} & NFN & 196.8 & - \\
\cline { 2 - 4 } & NF40 & 213.7 & 1.09 \\
\hline \multirow{2}{*}{$\begin{array}{c}\text { Series 2 } \\
\text { Shear Beams }\end{array}$} & NF100 & 227.2 & 1.15 \\
\cline { 2 - 4 } & NSN & 162.9 & - \\
\hline \multirow{2}{*}{$\begin{array}{c}\text { Series 3 } \\
\text { Bond Beams }\end{array}$} & NS100 & 162.8 & 0.99 \\
\cline { 2 - 4 } & NBN & 159.3 & 0.97 \\
\cline { 2 - 4 } & NB40 & 119.2 & - \\
\hline
\end{tabular}

* This is the average value of two replicate beams.

** This is the ratio of the ultimate load of beams with different percentage replacements of NCA with RCA relative to the control beam with 0 replacement in each series.

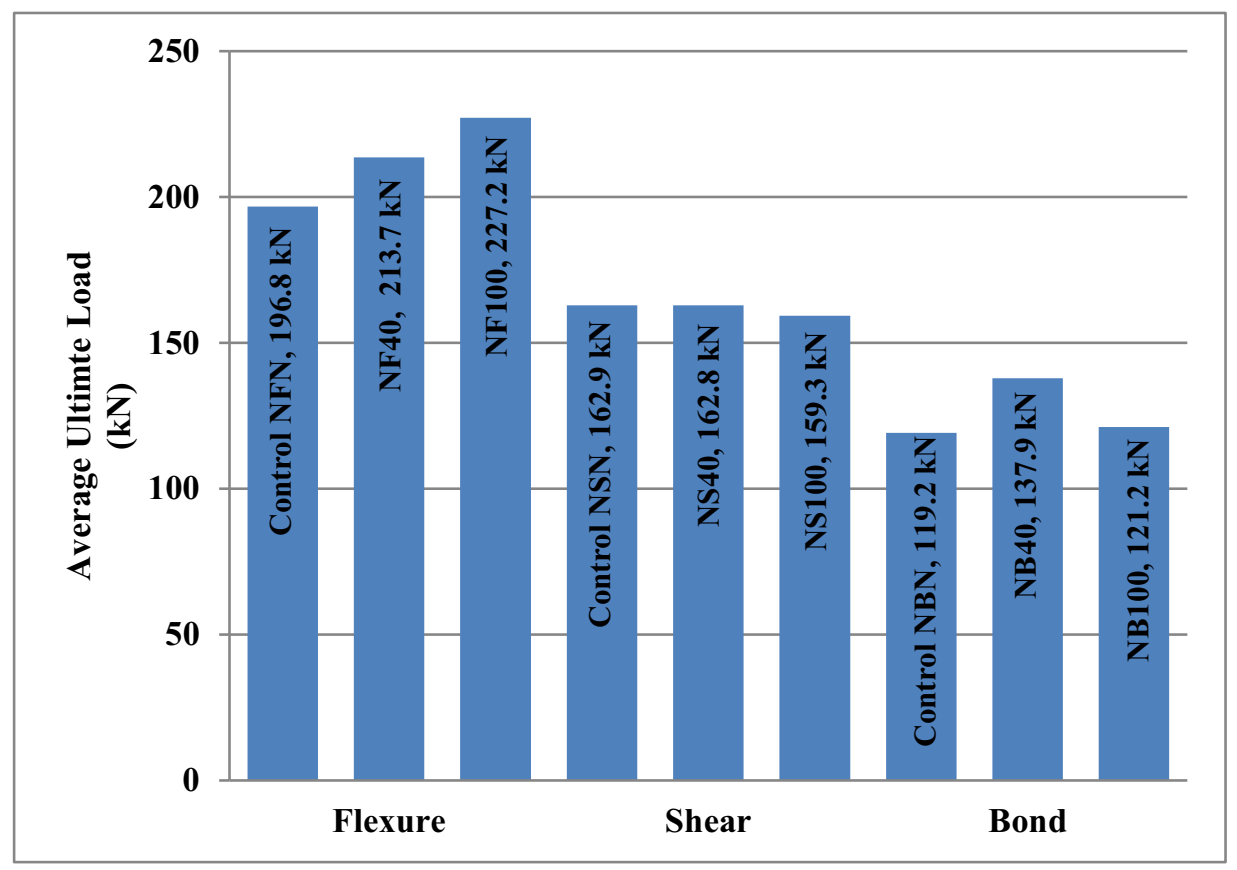

Fig. 5. Average ultimate loads for the beams in each series.

The ultimate loads of the 40 and the $100 \%$ replacement shear beam specimens were comparable to that of the control beam (Table 2 and Figure 5). Thus, the shear capacity was not affected by any percentage replacement of NCA with RCA. Also, the load-deflection histories of the shear beams with different percentage replacements were very similar with sharp drops in load after ultimate indicating brittle behavior. 
Failure of all bond beams occurred along the mid-span where the provided $305 \mathrm{~mm}$ splice length was less than the required design length. Bond splitting cracks propagated along the splice length on the side and on the bottom tension face of the beam. The average maximum loads were $119.2 \mathrm{kN}$ for the control shear beams, $137.9 \mathrm{kN}$ for the $40 \%$ replacement beams (16\% greater than the control beam), and $121.2 \mathrm{kN}$ for the $100 \%$ beams ( $2 \%$ greater than the control beam). Thus, the bond capacity was not negatively affected by any percentage replacement of NCA with RCA.

\section{Conclusion}

The paper reports on a two-phase research program conducted at the American University of Beirut.

The objective of the first phase was to study the effect of replacing part or all of the natural crushed limestone coarse aggregates (NCA) with recycled coarse aggregates (RCA) resulting from tested and crushed concrete cylinders in batching plants, on the plastic and hardened mechanical properties of the produced normal and high strength concrete mixes (NSC and HSC). Results indicated that for both types of concrete mixes with nominal strength values of 20 and $60 \mathrm{MPa}$, the replacement of NCA with different percentages of RCA $(0,20,40,60,80$, or $100 \%)$ did not have a negative effect on the consistency of fresh concrete. As for the NSC hardened concrete properties, average reductions of 9.8, 13.5, 11, and $9.2 \%$ were recorded relative to the control mix in the compressive strength, splitting tensile strength, modulus of elasticity, and flexural strength values, respectively. The average reductions in $\mathrm{HSC}$ were $11,11.3,10.3$, and 10.8\%, respectively. The reductions were not significantly affected by the percentage replacement of NCA with RCA or by the level of concrete strength.

For each of the three modes of failure, three beams with different percentages replacement of NCA with RCA [0 (control), 40, and 100 percent] were tested in the second phase. One replicate was prepared for each beam to validate the test results. Thus a total of 18 beams were tested with 6 for each mode of failure. The loading system produced a constant moment region at mid-span of the beam. Cylinder compressive strength tests indicated that mixes with $40 \%$ and $100 \%$ replacement percentages of NCA with RCA had reductions in the compressive strength relative to the mix with $0 \%$ replacement amounting to 9 and $11.7 \%$, respectively. However, the latter reduction in cylinder compressive strength did not have a significant impact on the mode of failure or ultimate strength or load-deflection behavior of reinforced concrete beams tested to fail in flexural or shear or bond splitting of the concrete cover in the splice region that could be related to the percentage replacement of NCA with RCA.

Results of the research program have a positive impact on the usage of recycled coarse aggregates produced by crushing tested concrete cylinders in the concrete construction industry. However, further research is needed to check the effect of recycled aggregate concrete sourced from tested concrete cylinders on other types of structural members such as columns and slabs.

\section{References}

1. ACI Report 555R-01. Removal and reuse of hardened concrete. ACI Committee 555, American Concrete Institute, Farmington Hills, Michigan (2010)

2. S. Nagataki, A. Gokce, T. Saeki, Effects of recycled aggregate characteristics on performance parameters of recycled aggregate concrete. ACI SP 192, American Concrete Institute, Farmington Hills, Michigan. 53-72 (2000) 
3. K. Rahal, Mechanical properties of concrete with recycled coarse aggregate. Build. \& Env. 42(1), 407-415 (2007)

4. M. Etxeberria, E. Vázquez, A. Marí, M. Barra. Influence of amount of recycled coarse aggregates and production process on properties of recycled aggregate concrete. Cem. \& Concr. Res. 37(5), 735-742 (2007)

5. K.H. Yang, H.S. Chung, A.F. Ashour. Influence of type and replacement level of recycled aggregates on concrete properties. ACI Mat. J. 105(3), 289-296 (2008)

6. ACI Committee 318. Building Code Requirements for Reinforced Concrete and Commentary (ACI-318-11/ACI-318R-11), American Concrete Institute, Farmington Hills, Michigan (2011)

7. S.F.U. Ahmed. Properties of concrete containing construction and demolition wastes and fly ash. ASCE J. of Mat. in Civ. Eng. 25(12), 1864-1870 (2012)

8. B.C. Han, H.D. Yun, S.Y. Chung. Shear capacity of reinforced concrete beams made with recycled-aggregate. ACI SP 200, American Concrete Institute, Farmington Hills, Michigan. 503-516 (2001)

9. F. Gholamreza, A.G. Razaqpur, O.B. Isgor, A. Abbas, B. Fournier, S. Foo. Flexural performance of steel-reinforced recycled concrete beam. ACI Str. J. 106(6), 858-867 (2009)

10. S.W. Kim, H.D. Yun. Influence of recycled coarse aggregates on the bond behavior of deformed bars in concrete. Eng. Str. 48, 133-143 (2012)

11. ACI Report 211.1-91. Standard practice for selecting proportions for normal, heavyweight, and mass concrete. ACI Committee 211, American Concrete Institute, Farmington Hills, Michigan (1991) 